\title{
ASO Author Reflections: Identification and Preservation of Axillary Lymphatic System
}

\author{
Qianqian Yuan, $\mathrm{MD}^{\mathbf{1}}$, and Gaosong $\mathrm{Wu}, \mathrm{MD}, \mathrm{PhD}^{\mathbf{1}}$ \\ Department of Thyroid and Breast Surgery, Zhongnan Hospital of Wuhan University, Wuhan, Hubei, People's Republic of \\ China
}

\section{PAST}

Application of axillary reverse mapping (ARM) in axillary dissection was introduced by Thompson from the USA and Nos from France with the aim of reducing the arm lymphedema rate after surgery for breast cancer. ${ }^{1,2}$ Several questions remain to be solved, including the unsatisfactory identification rate of arm nodes, uncertain oncological effect in long-term follow-up, and effectiveness in reducing lymphedema. ${ }^{3}$ The results of this clinical trial demonstrate that the refined ARM technique, DEPART, is effective and practical in eliminating arm lymphedema without compromising oncological safety.

\section{PRESENT}

Lymph from the arm is drained by 2-4 main afferents and then enters 1-2 nodes, that is, arm sentinel nodes. The key procedure, staged tracing (injection of $0.1 \mathrm{ml}$ blue dye into the arm sentinel nodes), enables visualization of the subsequent-echelon nodes and efferent lymphatics. The high detection rate $(81.0 \%)$ for the arm lymphatic system obtained in this study indicates that the DEPART technique was effective in the majority of patients. During the

This ASO Author Reflections is a brief invited commentary on the article "Identification and Preservation of Arm Lymphatic System in Axillary Dissection for Breast Cancer to Reduce Arm Lymphedema Events: a Randomized Clinical Trial," Ann Surg Oncol. (2019). h ttps://doi.org/10.1245/s10434-019-07569-4.

(C) Society of Surgical Oncology 2019

First Received: 19 September 2019;

Published Online: 30 October 2019

G. $\mathrm{Wu}, \mathrm{MD}, \mathrm{PhD}$

e-mail: wugaosongtj@163.com surgery, the gross arm nodes were examined by intraoperative partial frozen section and removed if positive. A 37-month follow-up revealed that this procedure reduced the arm lymphedema rate from $15.3 \%$ to $3.3 \%$ with nonsignificant difference in regional recurrence, which is of significance in improving postoperative quality of life of patients who undergo axillary dissection. ${ }^{4}$

\section{FUTURE}

This study had a single-surgeon design and was performed at two institutions. The DEPART technique is practical but requires a learning period to perform perfectly. Hence, its effectiveness in axillary dissection and the learning curve for the procedure require investigation in a future randomized multicenter study.

DISCLOSURES The authors report no conflicts of interest.

\section{REFERENCES}

1. Nos C, Lesieur B, Clough KB, Lecuru F. Blue dye injection in the arm in order to conserve the lymphatic drainage of the arm in breast cancer patients requiring an axillary dissection. Ann Surg Oncol. 2007;9:2490-96.

2. Thompson M, Korourian S, Henry-Tillman R et al. Axillary reverse mapping (ARM): a new concept to identify and enhance lymphatic preservation. Ann Surg Oncol. 2007;6:1890-95.

3. Beek MA, Gobardhan PD, Schoenmaeckers EJP et al. Axillary reverse mapping in axillary surgery for breast cancer: an update of the current status. Breast Cancer Res Treat. 2016;3:421-32.

4. Yuan Q, Wu G, Xiao S-Y, et al. Identification and preservation of arm lymphatic system in axillary dissection for breast cancer to reduce arm lymphedema events: a randomized clinical trial. Ann Surg Oncol. 2019. https://doi.org/10.1245/s10434-019-07569-4.

Publisher's Note Springer Nature remains neutral with regard to jurisdictional claims in published maps and institutional affiliations. 